\title{
Efeito da variação da espessura do revestimento nas respostas estruturais do pavimento flexível
}

\section{Effect of thickness variation of bituminous layer in the structural responses of flexible pavements}

\author{
Juliana Pavan Vidotto ${ }^{1}$; Heliana Barbosa Fontenele ${ }^{2}$
}

\section{Resumo}

Neste trabalho foi realizada uma análise de sensibilidade para investigar o efeito da variação da espessura do revestimento asfáltico nas respostas estruturais do pavimento flexível e, consequentemente, no seu desempenho. Para isso foram utilizados dados de tráfego provenientes de um posto de pesagem localizado no km 28 da Rodovia dos Imigrantes, no estado de São Paulo, dados esses coletados no ano de 2008. As cargas oriundas do tráfego foram aplicadas no programa ELSYM5 para a obtenção das respostas estruturais referentes aos defeitos fissuras por fadiga e afundamentos nas trilhas de rodas. Com esses valores, os fatores de dano foram calculados e assim a análise de sensibilidade, com relação à variação do parâmetro espessura do revestimento asfáltico do pavimento, foi realizada. Através da análise de todos os dados obtidos concluiu-se que a variação da espessura do revestimento asfáltico influencia nas respostas estruturais, sendo os fatores de danos referentes ao defeito fissura por fadiga mais sensíveis a tal variação que os fatores referentes ao defeito afundamento nas trilhas de roda.

Palavras-chave: Espessura do revestimento asfáltico. Respostas Estruturais. Deteriorações. Análise de Sensibilidade. ELSYM5.

\begin{abstract}
In this work, a sensitivity analysis was performed to investigate the effect of thickness variation of bituminous layer in the structural responses of flexible pavements and, therefore in performance. For this, it used traffic data from a weigh station located at $\mathrm{Km} 28$ of the Immigrants Highway, at state of Sao Paulo, whose data collection was realized in 2008. The loads deriving from traffic were applied to the computational program ELSYM5 to obtain structural responses related to damage such as fatigue cracks and rutting. With these values, the damage factors were calculated and then the sensitivity analysis related to the thickness variation of bituminous layer was performed. Through the obtained results was concluded that the variation of thickness of bituminous layer have an influence on structural responses, and that damage factors related to fatigue cracks were more sensitive than those factors related to rutting.
\end{abstract}

Key words: Thickness of bituminous layer. Structural Responses. Damage. Sensitivity Analysis. ELSYM5.

\footnotetext{
Graduada em Engenharia Civil, Universidade Estadual de Londrina; julianapavan@hotmail.com.br

2 Docente do Departamento de Construção Civil da Universidade Estadual de Londrina - DCCi-uel; heliana@uel.br
} 


\section{Introdução}

Odimensionamento da estrutura de um pavimento depende diretamente do tráfego nele existente. Nos últimos anos, o método de dimensionamento mais utilizado foi o empírico, que se baseia na experiência e na observação dos pavimentos. Os fatores utilizados variam de acordo com a região em que a rodovia é planejada, limitando a utilização de seus resultados com a eficiência desejada.

Estudos recentes vêm sendo desenvolvidos com a utilização do método empírico-mecanístico para dimensionar o pavimento. A base mecanicista utiliza métodos de cálculos mais precisos e realistas comparados ao método empírico, permitindo determinar as respostas estruturais do pavimento (tensão, deformação e deslocamento) utilizadas para analisar o desempenho do mesmo (FERNANDES JÚNIOR, 1994; FONTENELE, 2011).

A análise do pavimento através do método empírico-mecanístico parte de uma estrutura prédimensionada empiricamente. Com o auxílio de recursos computacionais, as respostas estruturais são determinadas e, através delas, calculam-se os danos acumulados durante a vida útil da rodovia. Isso permite estimar e comparar o comportamento do pavimento com os critérios de desempenho estabelecidos para a vida em serviço. Caso estes critérios não sejam obedecidos é necessário variar os parâmetros de projeto definidos até que a condição ideal seja obtida (FERNANDES JÚNIOR; PAES; PEREIRA, 2007; FONTENELE, 2011).

Em seu trabalho, Masad (2004) analisou o desempenho do pavimento através da variação dos parâmetros de projeto. Dessa forma, foi verificada a influência na formação das trilhas de roda e fissuras por fadiga no pavimento, utilizando as seguintes variáveis: clima, cargas de tráfego, espessura e material constituinte do revestimento asfáltico e material constituinte do subleito. Para a situação considerada no estudo, foi observado que as variações das propriedades e da espessura da base influenciaram diretamente na quantidade de fissuras que surgiam na rodovia. No caso da deformação nas trilhas de roda não houve quase nenhuma influência.

Em virtude do que foi mencionado, justificase então o estudo no tema proposto, voltado para a realidade brasileira, com o objetivo de verificar o efeito decorrente da variação da espessura do revestimento asfáltico nas respostas estruturais do pavimento e, consequentemente, no seu desempenho.

\section{Breve Abordagem Sobre o Dimensionamento de Pavimentos Flexíveis}

O dimensionamento dos pavimentos pode ser realizado com o uso de dois métodos: empírico e empírico-mecanístico. O método empírico é baseado unicamente em resultados experimentais de observação do desempenho dos pavimentos ao longo do tempo.

A abordagem empírica se restringe às condições em que foram desenvolvidos - fatores ambientais, tráfego dos veículos e propriedades dos materiais constituintes do pavimento - podendo o projeto não atingir sua eficiência máxima quando executado em diferentes condições ambientais e econômicas (GONÇALVES, 1999).

No dimensionamento empírico, o tráfego é convertido em cargas equivalentes ao eixo padrão através do número equivalente de solicitações do eixo padrão de 8,2 t (8.200 kg).

No Brasil, o método do DNER (atual DNIT) é o mais utilizado para projetos de pavimentos flexíveis. É um método baseado nos ensaios de CBR e nas curvas de dimensionamento do USACE (United States Army Corps of Engineers), considerando o conceito de equivalência estrutural estabelecido na pista experimental da AASHO (BEZERRA NETO, 2004).

Já o método empírico-mecanístico alia a base empírica com a base mecanicista. O pavimento é considerado como um sistema multicamadas 
onde se aplica os conceitos da Mecânica dos Pavimentos determinando as tensões, deformações e deslocamentos provenientes das cargas dos veículos. Esses valores são correlacionados com o desempenho do pavimento através de modelos empíricos de deteriorações.

O método mais recente desenvolvido para o dimensionamento empírico-mecanístico é o "Guia de Projetos AASHTO para o dimensionamento de estruturas de pavimentos novos e reabilitados" (o MEPDG - Mechanistic-Empirical Pavement Design Guide), desenvolvido através do Projeto NCHRP 1-37A com a pesquisa realizada pelo National Cooperative Highway Research Program (NCHRP) e patrocinada pela AASHTO. Nesse dimensionamento o tráfego é caracterizado através dos espectros de carga por eixo (Axle Load Spectra).

Os espectros de carga são distribuições de carga por eixo desenvolvidas para as configurações de eixo existentes (eixos simples, duplos, tandem duplos e triplos). Essas distribuições esboçam o tráfego de maneira mais fiel a existente na rodovia, já que todas as cargas por eixo são utilizadas (HAIDER; HARICHANDRAN, 2007).

De acordo com Jiang et al. (2008), o novo método de dimensionamento de pavimentos proposto pelo NCHRP é uma mudança radical do método empírico baseado nos ESALs (Equivalent Standard Axle Load), assim a partir da quantificação das características do tráfego usando diretamente todas as cargas por eixo, ao invés de convertê-las em ESALs, é possível analisar os impactos das variações das cargas de tráfego no pavimento e prover um adequado dimensionamento da estrutura do pavimento.

\section{Mecânica dos Pavimentos}

A mecânica dos pavimentos analisa o pavimento como um sistema em camadas sujeito à ação do carregamento aplicado pelos veículos. Essa análise permite determinar os parâmetros de deformabilidade (tensões, deformações e deslocamentos) que surgem em sua estrutura, conhecidos como respostas estruturais.

Muitos são os programas computacionais baseados na Teoria da Elasticidade de Sistemas Estratificados que calculam as respostas estruturais. Neste trabalho o cálculo será realizado com o uso do programa computacional ELSYM5, que utiliza o método das diferenças finitas.

Os dados de entrada utilizados nesse programa são: propriedades das camadas (espessura, módulo de elasticidade e coeficiente de Poisson), localização e magnitude das cargas e as coordenadas dos pontos para a determinação das respostas estruturais do pavimento - x, em direção perpendicular às cargas, e $y$, na direção paralela às cargas (FONTENELE, 2011).

\section{Mecanismos de Deterioração dos Pavimentos}

Os diversos modos de deterioração do pavimento podem surgir devido a fatores ambientais e da solicitação do tráfego existente na via, além de depender também do material que constitui a estrutura do pavimento.

Com a atuação das tensões provocadas pelo carregamento surgem as seguintes deformações na estrutura do pavimento: deformação horizontal de tração na fibra inferior do revestimento $\left(\varepsilon_{h t, 1}\right)$ e deformação vertical de compressão no topo do subleito $\left(\varepsilon_{1}(v c, m)\right)$.

\section{Fissuras por Fadiga}

A ação do carregamento induz tensões de tração e cisalhamento em todas as camadas do pavimento, provocando uma eventual perda de integridade estrutural em todas as camadas. As fissuras por fadiga iniciam-se nos pontos onde essas tensões de tração e cisalhamento atuam. Esses pontos são conhecidos como críticos. Uma vez iniciada as fissuras, a ação contínua do carregamento 
proporciona sua propagação através das camadas (NCHRP, 2004).

As fissuras por fadiga podem ser divididas em dois tipos: bottom-up e top-down. As fissuras bottom-up surgem na parte inferior do revestimento e se propagam até a superfície. Elas são causadas devido à ação de tensões de tração na fibra inferior do revestimento. Já as fissuras top-down iniciam-se no topo da camada de revestimento e se propagam para o interior do pavimento e surgem, possivelmete, devido às tensões de tração e cisalhamento atuantes na superfície do revestimento, causadas principalmente pelas altas pressões de enchimento dos pneus dos veículos (NCHRP, 2004).

Assim, é importante salientar que, em grande parte das análises, inclusive as apresentadas neste artigo, as tensões de tração são aquelas que produzem degradação por fadiga. Somente no caso específico das fissuras por fadiga do tipo top-down, as tensões de cisalhamento podem ser "as possíveis causadoras" de tal padrão de fissuração por fadiga, muito embora, conforme o próprio NCHRP (2004), tal observação é hipotética e com estudos inconclusivos. Nos modelos mais tradicionais de avaliação de fissuras por fadiga, o esforço único que gera a degradação por fadiga em revestimentos asfáltico é o de tração na flexão.

\section{Afundamento nas Trilhas de Rodas}

O afundamento nas trilhas de roda é um tipo de deformação permanente que ocorre quando existe o acúmulo de pequenas quantidades de deformações não recuperáveis, resultantes das aplicações de cargas pelos veículos, criando uma depressão longitudinal na superfície do pavimento (DNIT, 2006; NCHRP, 2004).

Com o aumento dos níveis de cargas e mudança nas características do tráfego, a tendência no dimensionamento é utilizar camadas de revestimentos mais espessas, consequentemente aumentando os problemas com afundamentos nas trilhas de rodas (FERNANDES JUNIOR; PAES; PEREIRA, 2007).

\section{Parâmetros de Projeto}

O dimensionamento empírico-mecanístico parte de uma estrutura pré-dimensionada. O projetista deve determinar uma seção transversal, muitas vezes com o auxílio do método empírico, e analisar se essa atende os critérios de desempenho solicitados no projeto. Quando esses critérios não são obedecidos os parâmetros utilizados devem ser modificados até obter um pavimento viável, tanto do ponto de vista estrutural quanto do funcional.

Os parâmetros utilizados no dimensionamento empírico-mecanístico são: carregamento do tráfego, propriedade dos materiais e espessura das camadas.

\section{Estudos sobre a Variação dos Parâmetros de Projeto}

Shahji (2006) avaliou a sensibilidade dos parâmetros de dimensionamento de pavimentos flexíveis e rígidos. $\mathrm{O}$ estudo foi baseado no MEPDG, utilizando informações contidas no Guia de Projetos, sobre as cargas de tráfego e sobre as propriedades dos materiais utilizados. A análise de sensibilidade foi realizada alterando um parâmetro de cada vez, mantendo todos os outros constantes. As conclusões obtidas por Shahji (2006) foram as seguintes:

- O aumento da espessura do revestimento acarreta em um aumento da formação das fissuras topdown;

- A variação da espessura do revestimento contribuiu para o aparecimento das fissuras bottom-up. Já as variações da espessura da base e da sub-base, além do módulo de elasticidade do subleito contribuíram pouco para o surgimento desse tipo de deterioração;

- O aumento da espessura do revestimento de 5,0 $\mathrm{cm}$ para $10 \mathrm{~cm}$ provoca um aumento na formação das fissuras bottom-up. Já com 
espessuras acima de $10 \mathrm{~cm}$ há uma diminuição nessa formação. Com isso, a melhor espessura para evitar o surgimento desse tipo de fissura é de $10 \mathrm{~cm}$ a $12,5 \mathrm{~cm}$;

- O afundamento em trilhas de rodas provém do acúmulo da deformação de cada camada e é mais afetado pela espessura do revestimento e pelas cargas de tráfego. Shahji (2006) verificou que, quando ocorre uma deformação permanente de 2,05 $\mathrm{cm}$ em todo o pavimento, $1,3 \mathrm{~cm}$ é acumulada pelo revestimento, $0,175 \mathrm{~cm}$ pelo base e $0,75 \mathrm{~cm}$ pelo subleito.

Outro estudo que avaliou os efeitos que a variação dos parâmetros de projeto proporciona nas respostas estruturais do pavimento foi o de Masad (2004). Neste trabalho também foi utilizado o MEPDG e as principais conclusões obtidas no estudo foram:

- A espessura e o módulo de elasticidade da base têm influência no surgimento das fissuras por fadiga;

- As propriedades da base não possuem quase nenhuma influência no surgimento dos afundamentos nas trilhas de rodas.

Schwartz et al. (2013) também realizaram uma análise de sensibilidade de pavimentos flexíveis para dados de projeto do MEPDG sob cinco condições climáticas e três níveis de tráfego. Os resultados encontrados apontam para o fato de que um dos dados de projeto mais consistentes nas categorias de sensibilidade para todos os defeitos foi a espessura do revestimento asfáltico. As fissuras por fadiga e as longitudinais também apresentaram sensibilidade com relação à espessura da base.

\section{Método de Pesquisa}

O estudo foi dividido em duas etapas: a primeira etapa consta da determinação: das respostas estruturais; dos fatores equivalentes de carga empírico-mecanísticos; dos fatores de veículos empírico-mecanísticos e do número equivalente de solicitações empírico-mecanísticos. A segunda etapa se referiu à análise de sensibilidade do pavimento.

Como abordado anteriormente este trabalho usou o ELSYM5 para o cálculo das deformações atribuídas ao surgimento dos defeitos fissuras por fadiga e afundamento nas trilhas de rodas. Para tanto foram adotados os espectros de carga por eixo, tendo em vista que atualmente é a forma de caracterização do tráfego utilizada pelo novo método de dimensionamento da AASHTO.

\section{Determinação do Tráfego}

Para a determinação das cargas de tráfego atuantes no pavimento foram utilizados os espectros de carga desenvolvidos por Fontenele (2011). Os dados relativos aos veículos utilizados para desenvolvimento desses espectros foram registrados no posto de pesagem PPV01, localizado no $\mathrm{km} \mathrm{28,} \mathrm{da} \mathrm{Rodovia} \mathrm{dos} \mathrm{Imigrantes.} \mathrm{Os} \mathrm{dados}$ foram coletados de janeiro a dezembro de 2008, com pesagens realizadas durante as 24 horas do dia.

Em seu trabalho, Fontenele (2011) utilizou os seguintes intervalos de carga estabelecidos no relatório NCHRP 1-37A:

- ESRS e ESRD: valores de carga entre 1.360,5 a $18.140,6 \mathrm{~kg}$, em intervalos de 453,5 kg (3.000 e $40.000 \mathrm{lbs}$, em intervalos de $1.000 \mathrm{lbs}$ );

- ETD: valores de carga entre 2.721,1 a 36.281,2 $\mathrm{kg}$, em intervalos de $907 \mathrm{~kg}$ (6.000 e $80.000 \mathrm{lbs}$, em intervalos de $2.000 \mathrm{lbs})$;

- ETT: valores de carga entre 5.442,2 a 46.258,5 $\mathrm{kg}$, em intervalos de $1.360,5 \mathrm{~kg}(12.000 \mathrm{a}$ $102.000 \mathrm{lbs}$, em intervalos de $3.000 \mathrm{lbs}$ ).

O tráfego considerado no estudo é composto de sete classes de veículos (Fontenele, 2011). Os tipos de veículos e suas respectivas freqüências na via são: $2 \mathrm{C}(8,72 \%) ; 3 \mathrm{C}(7,41 \%) ; 2 \mathrm{~S} 1(8,41 \%)$; 2S2 (11,18\%); 2S3 (27,4\%); 3S3 (13,32\%) e 3D4 $(23,56 \%)$. 
O VDMA do tráfego utilizado para o ano base de 2009 foi o fornecido pela Agência Reguladora de Serviços Públicos Delegados de Transportes do Estado de São Paulo (ARTESP), e obtido a partir de contagens na praça de pedágio do $\mathrm{km} \mathrm{32,381}$ (Piratininga - sentido Sul), da Rodovia SP 160, e por sensor de tráfego no sentido contrário, no Km 38,04 (Piratininga - sentido norte).

As respostas estruturais foram calculadas utilizando um pavimento pré-dimensionado empiricamente. Os valores da espessura, do módulo de elasticidade e do coeficiente de Poisson utilizados para cada camada são valores médios adotados no dimensionamento dos pavimentos brasileiros.

As características da estrutura do pavimento utilizado no estudo estão apresentadas na Tabela 1 .

Tabela 1 - Características do Pavimento.

\begin{tabular}{cccc}
\hline Camadas & $\begin{array}{c}\text { Espessura } \\
(\mathbf{c m})\end{array}$ & $\begin{array}{c}\text { Módulo } \\
\text { Elasticidade } \\
(\mathbf{M P a})\end{array}$ & $\begin{array}{c}\text { Coef. } \\
\text { Poisson }\end{array}$ \\
\hline 1 & 7,$5 ; 10,0 ;$ & 4.000 & 0,30 \\
2 & 12,5 e 15,0 & & 0,40 \\
3 & 20,0 & 400 & 0,40 \\
Subleito & 25,0 & 200 & 0,45 \\
\hline
\end{tabular}

Fonte: Vidotto (2012)

As análises foram realizadas utilizando as respostas estruturais referentes às deformações horizontais de tração na fibra inferior do revestimento e às deformações verticais de compressão vertical no topo do subleito. Elas foram obtidas com o uso do programa computacional de análises elásticolineares ELSYM5.

Os dados de entrada do programa são: propriedades das camadas constituintes do pavimento (espessura, coeficiente de Poisson, módulo de elasticidade), dados do carregamento (carga por roda e pressão de enchimento do pneu) e sua localização, e as coordenadas para determinação das respostas do pavimento (eixo $\mathrm{x}$, direção perpendicular ao tráfego, e eixo y, direção paralela ao tráfego).

As respostas estruturais foram determinadas no centro das superfícies de carregamento e em pontos equidistantes a ela, sendo $33 \mathrm{~cm}$ para o ESRD e de $120 \mathrm{~cm}$ para o ETD e ETT.

Para a obtenção das respostas estruturais referentes ao eixo padrão foi utilizada a pressão de enchimento de $552 \mathrm{kPa}$ (80 psi), carga por roda de $2050 \mathrm{~kg}$ e a localização de carga do ESRD. Já na obtenção das demais respostas estruturais foi utilizada uma pressão de enchimento de $827 \mathrm{kPa}$ (120 psi) e a carga correspondente ao ponto médio do intervalo de classe de cargas que representa o espectro de carga por eixo, dividida pelo número de rodas que compõem cada eixo. Essa pressão de enchimento de $827 \mathrm{kPa}$ (120 psi) é, de acordo com Fernandes Júnior, Paes e Pereira (2007), o valor médio utilizado no Brasil.

Nas Figuras 1 e 2 são apresentados, a título de exemplo, os espectros de carga por eixo desenvolvidos por Fontenele (2011) para o veículo 2S3.

Figura 1 - Espectro de carga por eixo para o ESRS do veículo $2 \mathrm{~S} 3$

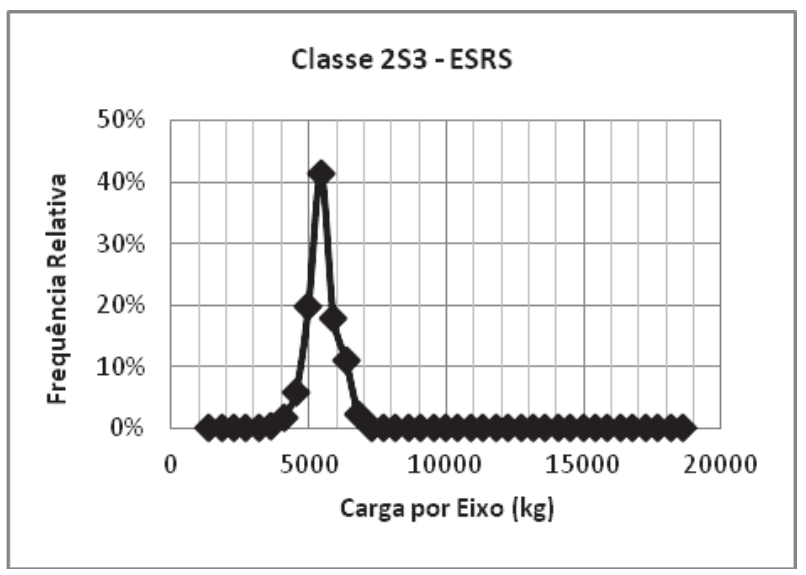

Fonte: Fontenele (2011) 
Figura 2 - Espectros de carga por eixo para o ESRD e o ETT do veículo $2 \mathrm{~S} 3$
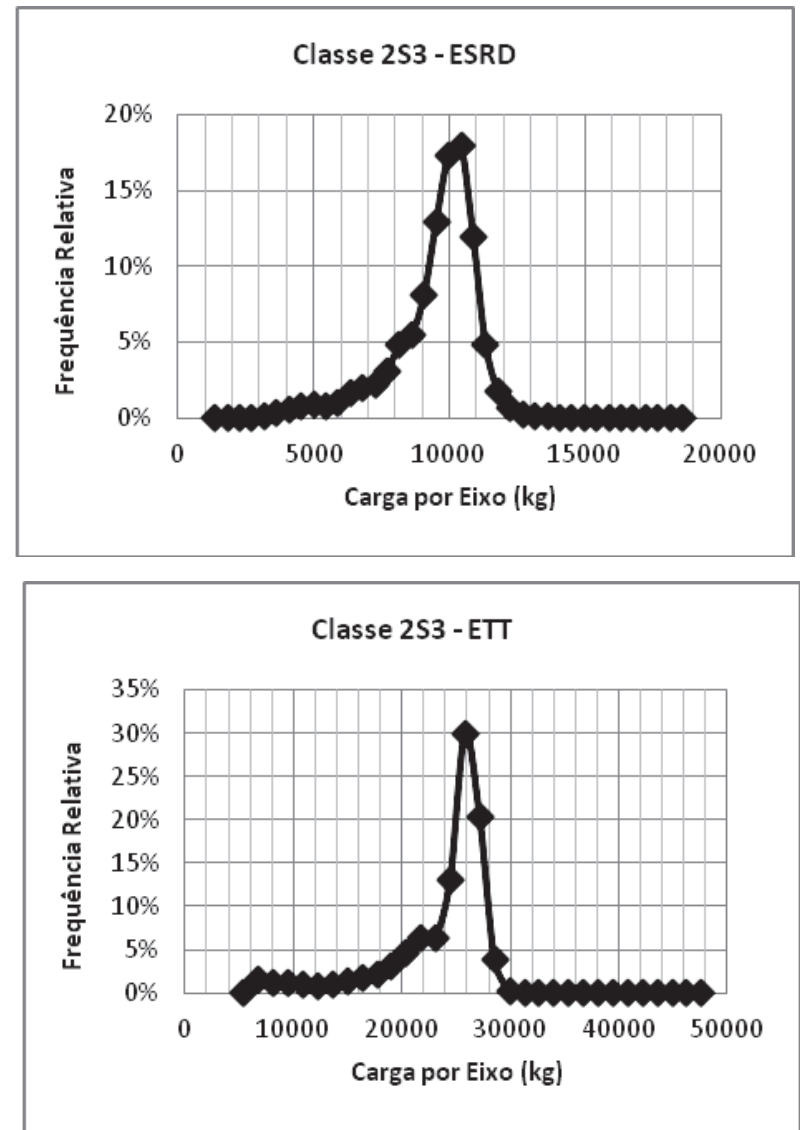

Fonte: Fontenele (2011)

\section{Cálculo dos Fatores de Danos Empírico- Mecanísticos}

Os fatores de equivalência de carga empíricomecanísticos (FEC) são relacionados aos danos provocados pela passagem de cada eixo do veículo na via. Eles foram obtidos para os dois tipos de deterioração em estudo: fissuras por fadiga e afundamento nas trilhas de roda.

O cálculo foi realizado com o uso da equação 1, obtendo o $\mathrm{FEC}_{\text {fissuras }}$ (utilizando a deformação horizontal de tração na fibra inferior do revestimento $-\varepsilon_{h t, 1}$ ) e $\mathrm{FEC}_{\text {afundamento }}$ (utilizando a deformação vertical de compressão no topo do subleito $-\varepsilon_{v c, m}$ ) com o uso dos expoentes 3,291 e 4 , respectivamente. Esses expoentes são os mesmo utilizados por Fontenele (2011) em seu trabalho e eles são relacionados com forma de deterioração, à resposta estrutural e ao tipo de estrutura analisada.

$$
F E C_{i}=\left(\frac{\rho_{i}}{\rho_{o}}\right)^{b}
$$

Onde:

$\rho_{i}=$ Resposta estrutural correspondente à solicitação em análise;

$\rho_{o}=$ Resposta estrutural correspondente à solicitação padrão;

$b=$ Expoente associado à forma de deterioração, à resposta estrutural considerada, ao tipo de ensaio e à estrutura analisada;

A partir da somatória dos FECs de cada eixo que compunha cada classe de veículo foi calculado o valor do fator de veículo para cada classe individualmente (FV) para de acordo com os dois tipos de deterioração $\left(\mathrm{FV}_{\text {fissuras }}\right.$ e $\left.\mathrm{FV}_{\text {afundamento }}\right)$. Posteriormente, os FV calculados para cada classe individual de veículos foram ponderados de acordo com sua frequência na via, ou seja, foram multiplicados pela frequência relativa de cada classe de pesagem, obtendo-se, a partir da somatória de todos os valores, o FV médio da frota.

\section{Cálculo do N Empírico-Mecanístico}

Para a determinação do valor de "N" para o período de projeto de 20 anos foi calculado, inicialmente, o VDMA para cada ano, dentro do referido período, usando-se a Equação 2, que considera uma projeção linear do tráfego. $\mathrm{O}$ valor de "N" relativo a cada ano foi obtido a partir da Equação 3, sendo posteriormente acumulado até o $20^{\circ}$ ano, Equação 4.

$$
\begin{gathered}
V_{n}=V_{o}+n \times t \\
N_{a}=365 \times F V \times V_{n} \times c
\end{gathered}
$$




$$
N=\sum_{a=1}^{a=p} N_{a}
$$

onde:

$V_{n}:$ volume de tráfego no ano "n";

$V_{0}:$ volume de tráfego no ano base;

n: número de anos decorridos após o ano base;

$t$ : taxa de crescimento anual;

$N_{a}$ : número equivalente de solicitações durante o ano "a";

$\boldsymbol{F V}$ : fator de veículos;

c: fator de distribuição de veículos comerciais em relação ao tráfego comercial, nos dois sentidos, na faixa de projeto;

$\mathbf{N}$ : número equivalente de solicitações durante o período de projeto;

$a$ : ano do período de projeto;

p: número de anos do período de projeto.

Para o cálculo foram considerados a taxa anual de crescimento do tráfego de $4 \%$ ao ano e o "c" igual a $48 \%$, pois a rodovia é de pista dupla com quatro faixas por sentido.

No cálculo de N, foi usada a equação 3 aplicandose os valores de $\mathrm{FV}_{\text {fissura }}$ e $\mathrm{FV}_{\text {afundamento }}$ médios da frota. Com isso foi obtido o consumo da vida de projeto do pavimento, em termos das solicitações correspondentes aos efeitos de deterioração causados pelos dois tipos de deformações consideradas na pesquisa.

\section{Análise de Sensibilidade}

Os valores dos fatores de equivalência de cargas empírico-mecanístico, dos fatores de veículos e dos números de solicitações "N" para o último ano de projeto, determinados a partir das respostas estruturais, foram relacionados graficamente com a variação das espessuras do revestimento do pavimento flexível.

\section{Apresentação e Análise de Resultados}

Fator de Equivalência de Carga (FEC) EmpíricoMecanístico

Os resultados obtidos para os valores de FEC de cada eixo dos veículos foram relacionados graficamente de acordo com a variação da espessura do revestimento asfáltico.

Para fins de praticidade, o gráfico apresentado é referente ao veículo $2 \mathrm{~S} 3$, sendo este o de maior frequência na via, demonstrando a influência que a variação da espessura provoca nos valores de FEC.

$\mathrm{O}$ veículo de classe $2 \mathrm{~S} 3$ apresenta uma frequência de $27,40 \%$ na via, sendo o maior valor observado. O gráfico relacionando os valores de FEC de cada um dos eixos com as diferentes espessuras do revestimento flexível encontra-se na Figura 3.

Figura 3 - Fatores de equivalência de carga empíricomecanístico para o veículo $2 \mathrm{~S} 3$

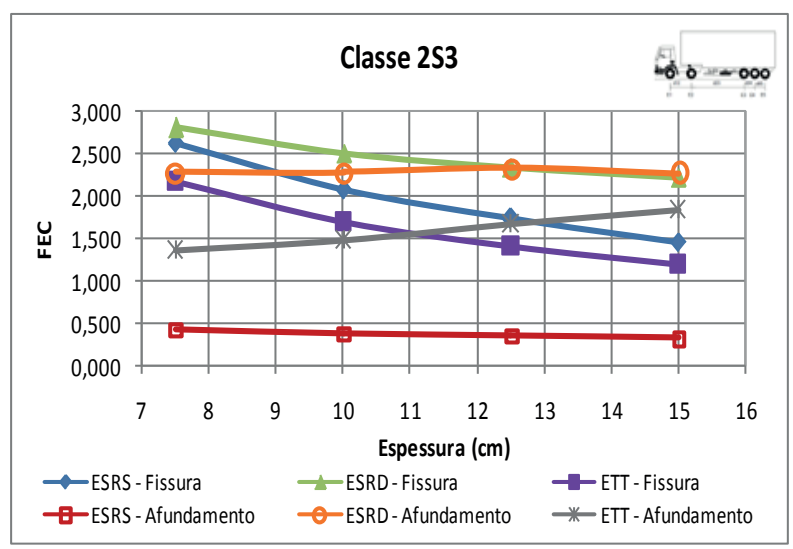

Fonte: Vidotto (2012)

Observando a Figura 3, nota-se que os valores dos FEC possuem diferentes comportamentos para cada tipo de eixo do veículo, assim as análises foram feitas separadamente.

Os valores dos FEC para o ESRS, relativos ao defeito fissura, diminuem $44,25 \%$ com o aumento de espessura do revestimento. Já os valores do FEC para o ESRS relativo ao defeito afundamento 
possuem uma variação menor comparado ao defeito fissura, diminuindo $24,1 \%$.

Quanto maior o valor do FEC, mais danosa é a passagem do eixo no pavimento. Com isso, na situação em estudo e considerando este tipo de eixo, é viável o aumento da espessura do revestimento para o controle dos dois tipos de defeitos. Para a deterioração fissura por fadiga a variação é mais acentuada, ou seja, o ESRS possui maior sensibilidade no que tange ao defeito fissura por fadiga, pois esse sofre maiores variações no valor do FEC.

Para o ESRD, os valores do FEC relativos ao defeito fissura seguem o mesmo comportamento do ESRS, ou seja, o FEC diminui à medida que a espessura aumenta em 20,74\%.Os valores do FEC para o ESRD relativo ao defeito afundamento possui um comportamento diferenciado aumentando $1,89 \%$ entre as espessuras de $7,5 \mathrm{~cm}$ e $12,5 \mathrm{~cm}$, e diminuindo $2,44 \%$ da espessura de $12,5 \mathrm{~cm}$ para $15 \mathrm{~cm}$. A variação da espessura de $7,5 \mathrm{~cm}$ para 15 cm é de $0,57 \%$, ou seja, é uma diminuição pouco acentuada.

Essa variação no valor do FEC mostra que, para o ESRD, o FEC referente ao defeito fissura é mais sensível à variação de espessura do que o mesmo fator referente ao defeito afundamento nas trilhas de rodas. Com isso, na situação em estudo e para o ESRD verifica-se que é viável o aumento da espessura para o controle das fissuras por fadiga. Já com relação ao afundamento nas trilhas, de forma geral a sensibilidade para este defeito é pequena, não contribuindo significativamente.

Os valores dos FEC para o ETT relativos ao defeito fissura por fadiga diminuem em $44,75 \%$ com o aumento da espessura de $7,5 \mathrm{~cm}$ para $15 \mathrm{~cm}$. Já os valores dos FEC para o ETT relativos ao defeito afundamento aumentam $26 \%$ na mesma variação de espessura. Essa diferença de porcentagem mostra que o FEC referente ao defeito fissura por fadiga é mais sensível que o FEC referente ao defeito afundamento, para o eixo em questão. Com isso, para este tipo de eixo verifica-se um comportamento oposto com relação ao dano relativo aos dois tipos de defeito, sendo que para as fissuras por fadiga é interessante o aumento da espessura e para o afundamento esse aumento contribui negativamente.

Na Tabela 2 estão os valores de FEC obtidos a partir da simulação com a espessura do revestimento asfáltico igual a $7,5 \mathrm{~cm}$.

Tabela 2 - Valores de FEC com a adoção 7,5 $\mathrm{cm}$ de espessura de revestimento.

\begin{tabular}{|c|c|c|c|}
\hline Classe & Tipo Eixo & FEC $_{\text {fissura }}$ & FEC $_{\text {afundamento }}$ \\
\hline \multirow{2}{*}{$2 \mathrm{C}$} & ESRS & 1,518 & 0,118 \\
\hline & ESRD & 1,261 & 0,520 \\
\hline \multirow{3}{*}{$2 \mathrm{~S} 1$} & ESRS & 2,199 & 0,231 \\
\hline & ESRD 1 & 1,099 & 0,263 \\
\hline & ESRD 2 & 2,026 & 0,967 \\
\hline \multirow{3}{*}{$2 \mathrm{~S} 2$} & ESRS & 2,287 & 0,269 \\
\hline & ESRD & 1,480 & 0,602 \\
\hline & ETD & 0,846 & 0,228 \\
\hline \multirow{3}{*}{$2 \mathrm{~S} 3$} & ESRS & 2,637 & 0,432 \\
\hline & ESRD & 2,813 & 2,290 \\
\hline & ETT & 2,177 & 1,370 \\
\hline \multirow{2}{*}{$3 \mathrm{C}$} & ESRS & 2,288 & 0,325 \\
\hline & ESRD & 1,670 & 0,845 \\
\hline \multirow{4}{*}{$3 \mathrm{D} 4$} & ESRS & 2,897 & 0,601 \\
\hline & ETD 1 & 2,458 & 1,591 \\
\hline & ETD 2 & 2,428 & 1,536 \\
\hline & ETD 3 & 2,403 & 1,495 \\
\hline \multirow{3}{*}{$3 \mathrm{~S} 3$} & ESRS & 2,676 & 0,458 \\
\hline & ETD & 2,059 & 1,068 \\
\hline & ETT & 2,304 & 1,469 \\
\hline
\end{tabular}

Fonte: Vidotto (2012)

A partir de uma rápida observação da Tabela 2 percebe-se o efeito danoso do ESRS relacionado às fissuras por fadiga. Na maioria das vezes ele é superior ao dano causado pelos outros tipos de eixo. 
Esta observação deixa clara uma das limitações do método empírico de dimensionamento, que considera de forma equivalente os efeitos dos eixos simples, ou seja, efeitos iguais tanto para aqueles eixos de rodado simples como duplo.

\section{Fator de Veículo Empírico-Mecanístico Médio da Frota}

Com os valores do FEC empírico-mecanísticos determinado para cada tipo de eixo foi possível obter os valores do fator de veículo empírico-mecanístico (FV) para cada classe de veículo. Esse fator indica o dano médio que cada veículo, individualmente, provoca ao circular na via em termos equivalentes ao eixo padrão. Assim calculou-se o fator de veículo empírico-mecanístico médio da frota $(\overline{\mathrm{FV}})$ a partir da consideração da freqüência de cada classe na via.

A Figura 4 mostra o gráfico que foi gerado utilizando os valores do fator de veículo médio da frota com a variação das espessuras do revestimento do pavimento. Pode-se observar que as curvas relacionadas à fissura por fadiga e ao afundamento nas trilhas de roda possuem direções opostas. No caso do $\overline{\mathrm{FV}}$ relativo ao defeito fissura há uma variação de $41 \%$ quando a espessura é aumentada de $7,5 \mathrm{~cm}$ para $15 \mathrm{~cm}$. Já no defeito afundamento a variação é de $9 \%$, sendo 4,5 vezes menor que no defeito citado anteriormente. Logo, o $\overline{\mathrm{FV}}$ é sensível à variação de espessura do revestimento asfáltico, sendo que a sensibilidade é maior para o defeito fissura por fadiga.
Figura 4 - Fator de veículo empírico-mecanístico médio da frota.

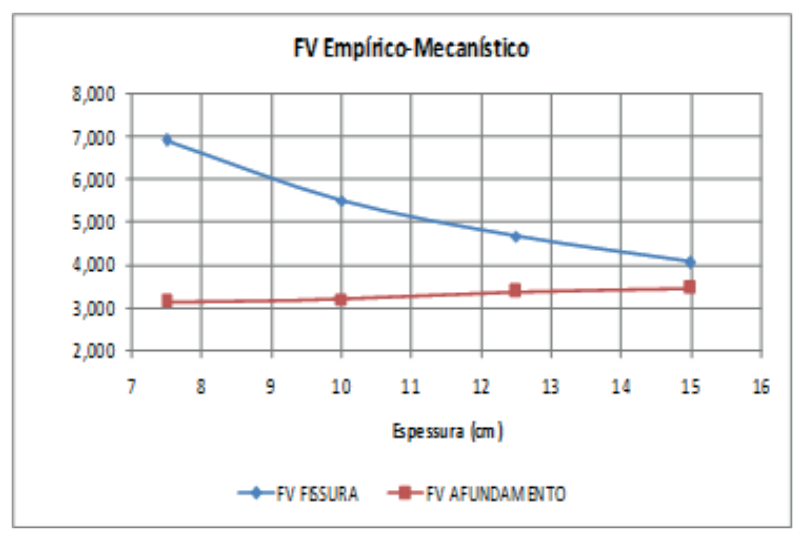

Fonte: Vidotto (2012)

\section{Número Equivalente de Solicitações Empírico-} Mecanístico

Utilizado os fatores de veículos obtidos foi possível determinar o número de solicitações acumulado ao longo do período de projeto $(\mathrm{N}$ empírico-mecanístico). Esse número indica o consumo do pavimento durante sua vida útil. Para facilitar a visualização foi gerado um gráfico com os resultados de $\mathrm{N}$ empírico-mecanístico para o último ano de projeto, ou seja, o ano de 2028, como indicado na Figura 5.

Analisando a Figura 5 nota-se que o valor de $\mathrm{N}$ empírico-mecanístico relativo ao defeito fissura diminui 41,3\% quando há variação da espessura de $7,5 \mathrm{~cm}$ para $15 \mathrm{~cm}$. Isso significa que com o aumento da espessura o número de passagens do eixo padrão para o mesmo dano diminui, ou seja, como não há variação do tráfego, o número de passagens dos veículos na rodovia é constante e o dano provocado por ele é menor. No caso do afundamento na trilha de roda, há um aumento de $9 \%$ no valor de $\mathrm{N}$ empírico-mecanístico quando relativo a esse defeito. Esse aumento não é considerado tão representativo comparado à variação observada no $\mathrm{N}$ empíricomecanístico relativo ao defeito fissura por fadiga. 
Figura 5 - Número de Solicitações Empírico-Mecanístico para o ano de 2028.

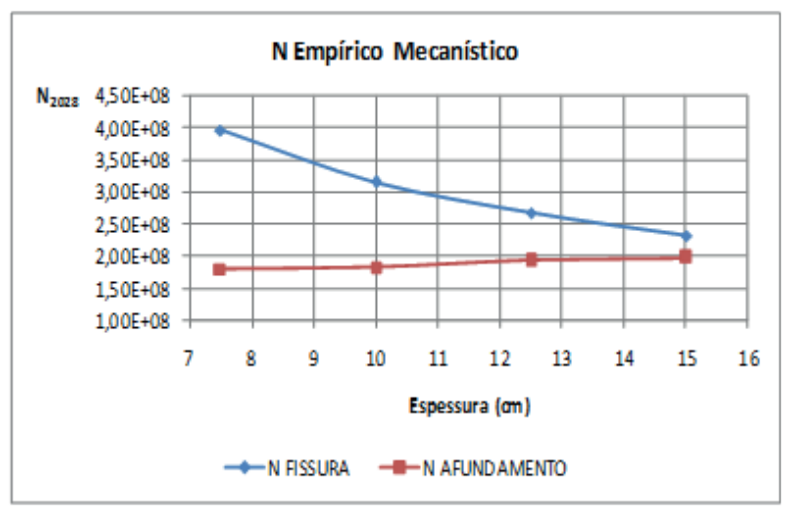

Fonte: Vidotto (2012)

\section{Conclusões}

Com a análise do efeito que a variação da espessura provoca nas respostas estruturais do pavimento flexível verificou-se que, para o fator de equivalência de carga empírico-mecanístico, a maior sensibilidade ocorre no defeito relativo às fissuras por fadiga.

Já na análise do fator de veículo empíricomecanístico médio da frota foi verificado que o valor do $(\overline{\mathrm{FV}})$ para o defeito fissuras por fadiga diminui $41 \%$ quando há variação da espessura de $7,5 \mathrm{~cm}$ para $15 \mathrm{~cm}$. No caso do defeito afundamento nas trilhas de roda, o valor do $(\overline{\mathrm{FV}})$ aumenta $9 \%$ na mesma variação de espessura. Logo, o defeito fissuras por fadiga é 4,5 vezes mais sensível a variação comparado ao defeito afundamento.

O número equivalente de solicitações empíricomecanístico apresenta a mesma variação que o $(\overline{\mathrm{FV}})$. No caso do defeito fissuras por fadiga, o valor do $\mathrm{N}$ diminui conforme a espessura é aumentada, logo o consumo do pavimento diminui com o aumento da espessura. Já para o defeito afundamentos nas trilhas de rodas, o comportamento é o contrário, aumentando o valor do $\mathrm{N}$ com o aumento da espessura, mas esse aumento não é considerado significativo, do mesmo modo que na sensibilidade do fator de veículo médio da frota.
Diante do exposto, em termo de variação de espessuras é possível constatar que a espessura do revestimento foi o parâmetro que mostrou ter o maior efeito, principalmente no que se refere ao defeito fissuras por fadiga. Confirmam-se, assim, as evidências de estudos anteriores, inclusive os que levaram à adoção, há muito tempo, por parte da AASHTO (1986), de espessura mínima de 12,5 cm para o revestimento asfáltico de rodovias com tráfego médio, ou seja, correspondente a um número de aplicações do eixo-padrão relativamente baixo ( $>5$ $\mathrm{x} 10^{6}$ ). Infelizmente, no Brasil, todas as evidências relacionadas à importância da espessura da camada de revestimento asfáltico têm sido ignoradas, com adoção de valores que, se representam alguma economia no custo de construção, resultam em custos altíssimos de manutenção e reabilitação e, mais ainda, de operação de veículos ao longo da vida em serviço.

\section{Agradecimentos}

Os autores agradecem à Fundação Araucária pelo auxílio financeiro fornecido à pesquisa de Protocolo $n^{\circ} 24685$, aprovada na Chamada 14/2011, que possibilitou a elaboração deste trabalho.

\section{Referências}

AMERICAN ASSOCIATION OF STATE HIGHWAY AND TRANSPORTATION OFFICIALS - AASHTO. Guide for design of pavement structures. Washington, D. C., 1986.

BEZERRA NETO, R. S. Análise comparativa de pavimentos dimensionados através dos métodos empirico do DNER e mecanístico e proposta de um catálogo simplificado de pavimentos para a região de Campo Grande (MS). 2004. Tese (Mestrado) - Pós-graduação em Engenharia Civil - Escola de Engenharia de São Carlos, Universidade de São Paulo, São Paulo.

DEPARTAMENTO NACIONAL DE INFRAESTRUTURA DE TRANSPORTE - DNIT. Manual de restauração de pavimentos asfálticos. Pub. IPR-720. Rio de Janeiro, 2006. 
FERNANDES JÚNIOR, J. L. Investigação dos Efeitos das Solicitações do Tráfego sobre o Desempenho de Pavimentos. 1995. 293 f. Tese (Doutorado) - Pósgraduação em Engenharia Civil - Escola de Engenharia de São Carlos, Universidade de São Paulo, São Carlos, São Paulo, 1994.

FERNANDES JÚNIOR, J. L.; PAES, J. C.; PEREIRA, P. A. A. Avaliação dos efeitos das solicitações do tráfego sobre o desempenho de pavimentos flexíveis portugueses e brasileiros. Revista Pavimentação, Rio de Janeiro, Ano II, n. 5, p. 28-38, 2007.

FONTENELE, H. B. Representação do tráfego de veículos rodoviários de carga através de espectros de carga por eixo e seu efeito no desempenho dos pavimentos. 2011. 287 p. Tese (Doutorado). Escola de Engenharia de São Carlos, Universidade de São Paulo, São Carlos.

GONÇALVES, F. J. P. O desempenho dos pavimentos flexíveis. 1999. Disponível em <http://usuarios.upf. br/ pugliero/arquivos/09.pdf $>$. Acesso em: 31 jun. 2012.

HAIDER, S. W.; HARICHANDRAN, R. S. Characterizing axle load spectra by using gross vehicle weights and truck traffic volumes. Transportation Research Record: Journal of the Transportation Research Board. TRB 2007 Annual Meeting CD-ROM. Washington, D. C., 2007.

JIANG, Y.; LI, S.; NANTUNG, T.; MANGOLD, K.; MacARTHUR, S. A. Creation of Truck Axle Load Spectra Using Weigh-in-motion Data. Journal of the transportation Research Forum, vol. 47, n. 4, pp. 45-61. Transportation Research Forum, 2008.
MASAD, S. Sensitivity Analysis of Flexible Pavement Response and AASHTO 2002 Design Guide for Properties of Unbound Layers. 2004. Thesis (Master of Science). Texas A\&M University.

NATIONAL COOPERATIVE HIGHWAY RESEARCH PROGRAM - NCHRP. Guide for Mechanistic-Empirical Design of New and Rehabilitated Pavement Structures. Final Report NCHRP 1-37 A. Champaign, Illinois, 2004.

SCHWARTZ, W. C.; LI, R.; CEYLAN, H.; KIM, S.; GOPALAKRISHNAN, K. Global Sensitivity Analysis of Mechanistic-Empirical Performance Predictions for Flexible Pavements. Transportation Research Board. TRB 2013 Annual Meeting CD-ROM. Washington, D. C. 2013.

SHAHJI, S. Sensitivity analysis of AASHTO'S 2002 flexible and rigid pavement design methods. 2006. Thesis (Master of Science). University of Central Florida, 2006.

VIDOTTO, J. P. Efeito da variação da espessura do revestimento nas respostasestruturais do pavimento flexível. 2012. 80 p. Trabalho de Conclusão de Curso (Graduação). Universidade Estadual de Londrina, Londrina. 